\title{
Autoinducer-Fluorophore Conjugates Enable FRET in LuxR Proteins in Vitro and in Cells
}

\author{
Matthew J. Styles, Michelle E. Boursier, ${ }^{\dagger}$ Margaret A. McEwan, ${ }^{\ddagger}$ Emma E. Santa, \\ Margrith E. Mattmann, $\S^{\S}$ Betty L. Slinger, and Helen E. Blackwell*
}

Department of Chemistry, University of Wisconsin-Madison, 1101 University Ave., Madison, WI 53706, USA; blackwell@chem.wisc.edu

\section{ABSTRACT}

Cell-to-cell signaling, or quorum sensing (QS), in Gram-negative bacteria is governed by small molecule signals ( $\mathrm{N}$-acyl L-homoserine lactones, AHLs) and their cognate intracellular receptors (LuxR-type proteins). The mechanistic underpinnings of QS in these bacteria are severely limited due to the challenges of isolating and manipulating most LuxR-type proteins.

Quantitative assays to characterize the direct binding of ligands to these receptors are largely non-existent. We report herein a Förster Resonance Energy Transfer (FRET) assay that leverages (i) conserved endogenous tryptophans located in the LuxR-type protein ligandbinding site and synthetic fluorophore-AHL conjugates, and (ii) isolation/stabilization of the proteins bound to weak agonists. The FRET assay permits straightforward measurement of ligand-binding affinities with receptor-either in vitro or in cells-and was shown to be compatible with six LuxR-type receptors. These methods will advance fundamental investigations of the mechanisms of LuxR-type proteins and the development of small molecule modulators of QS.

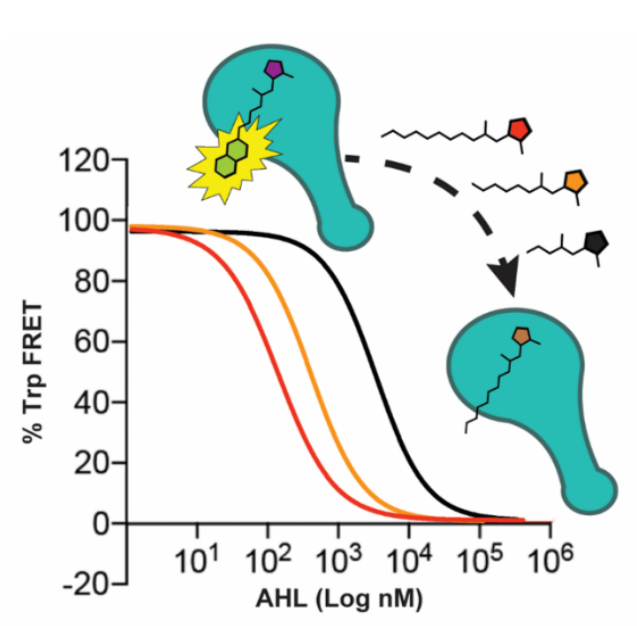

\footnotetext{
${ }^{+}$Current address: Amgen, Inc., 1 Amgen Center Dr., Thousand Oaks, CA 91320

${ }^{\ddagger}$ Current address: Labcorp Drug Development, 3301 Kinsman Blvd., Madison, WI 53704

$\S$ Current address: Truvian, 10300 Campus Point Dr., San Diego, CA 92121
} 


\section{INTRODUCTION}

Many common bacteria regulate important phenotypes in a population dependent manner through a cell-to-cell signaling mechanism called quorum sensing (QS). ${ }^{1,2}$ During QS, the bacteria produce a small molecule or short peptide that accumulates in the environment in proportion to the population density of the bacteria producing this signal. ${ }^{1}$ Once the signal reaches a threshold concentration in a given environment, a signal:receptor binding event triggers the bacteria to alter gene expression in order to initiate beneficial behaviors that are often only possible as a group. N-acyl L-homoserine lactones (AHLs) are one of the primary QS signal molecules used by Gram-negative bacteria. ${ }^{1} \mathrm{AHL}$-based QS is mediated by the canonical LuxI/LuxR synthase/receptor system, first described in the bioluminescent symbiont Vibrio fischeri (Fig. 1A). ${ }^{2}$ The Luxl-type synthase produces the AHL signal, and the cognate intracellular LuxR-type protein selectively binds to that AHL once a sufficient concentration, and thus quorate cell density, has been achieved. LuxR-type receptors are transcription factors that regulate gene expression; AHL binding can mediate either association of the LuxR-type receptor with DNA (for the more common activator class) or its disassociation from DNA (for the more rare repressor class). ${ }^{3}$ 


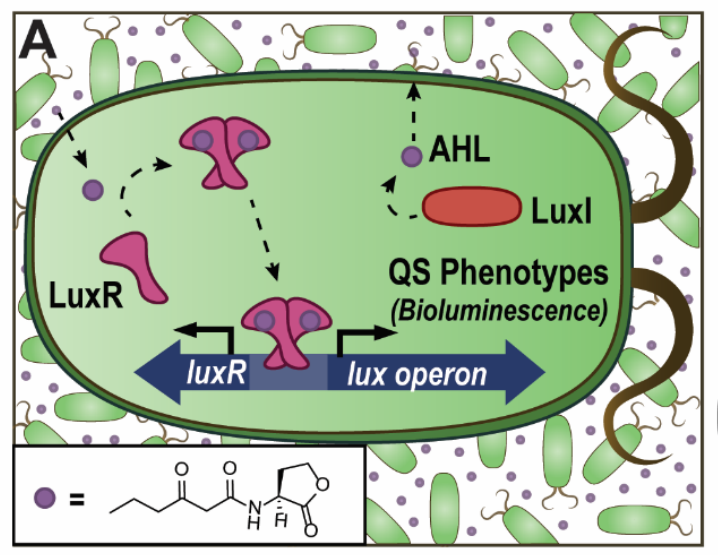

C 62

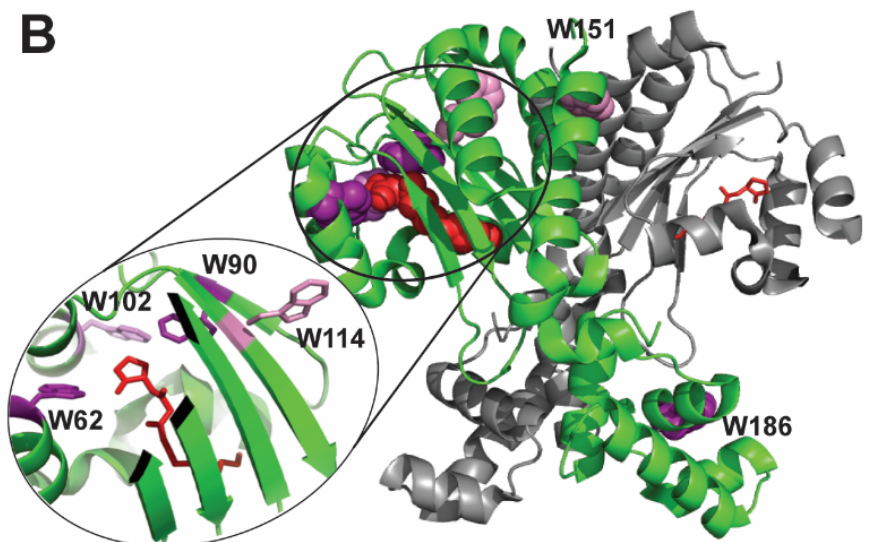

90 102 114 186

QSCR M...SNYPGEWKSRYISEDYTSIDPIVRHGLLEYMPLIWNGE---DF--QENRFFWEEALH--HGIRHGWS...TARETEMLKWTAVGK...

LasR M...GNYPAAWREHYDRAGYARVDPTVSHCTQSVLPIFWEPS---IYQTRKQHEFFEEASA--AGLVYGLT...TSREKEVLLWCAIGK...

RhIR M...GTYPKAWLERYQMQNYGAVDPAILNGLRSSEMVVWSDS---LF--DQSRMLWNEARD--WGLCVGAT...SHREREILQWTADGK...

SdiA $_{\text {eC }}$ M...TNYPESWVSYYQAKNFLAIDPVLNPENFSQGHLMWNDD---LF--SEAQPLWEAARA--HGLRRGVT...SKREKEILKWTAEGK...

SdiA $_{\text {se }}$ M...TTYPPAWVTHYQSENYFAIDPVLKPENFRQGHLHWDDV---LF--HEAKAMWDAAQR--FGLRRGVT...SKREKEILKWTAEGK...

CVIR M...VSYPSDWLDQYMKENYAQHDPILRI-HLGQGPVMWEER-FNRAKGAEEKREIAEATQNGMGS--GIT...SQREYDIFHWMSRGK...

TraR M...TNYHHDWRSLYFDKKFDALDPVVKRARSRKQVFAWSGEQERPALSKEERAFYAQAAD--FGIRSGIT...DPKEATYLRWIAVGK...

LUXR M...DNYPKKWRRQYYDDANLIKYDPIVDYSNSNHSPINWNIFE-NNAVNKKSPNVIKEAKT--SGLITGFS...TKREKECLAWACEGK...

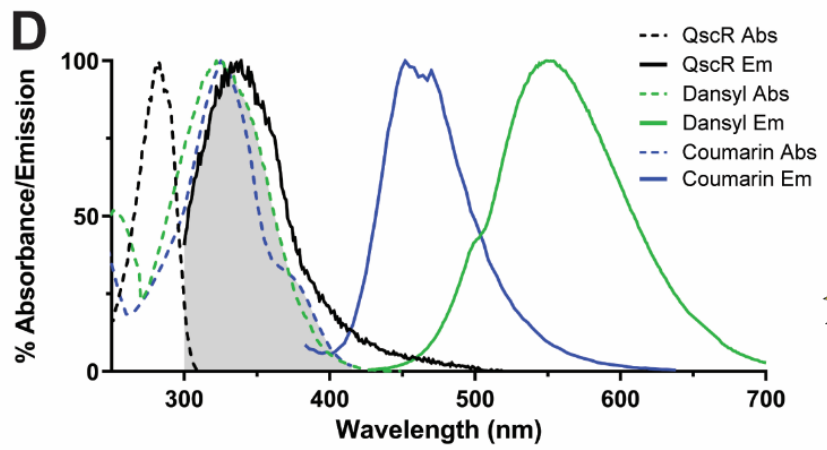

E

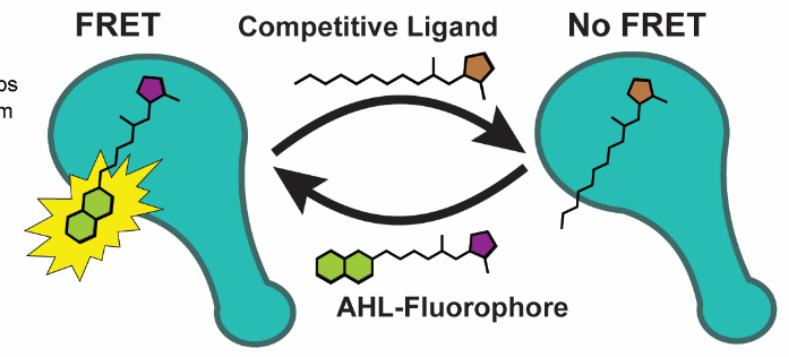

Figure 1. A) The canonical AHL-mediated LuxI/LuxR-type QS system from V. fischeri. Luxl produces $N$ (3-oxo)-hexanoyl L-homoserine lactone (OHHL), which passively diffuses from the cell and into others. At high population density, the concentration of intracellular $\mathrm{OHHL}$ is sufficiently high to bind to and stabilize LuxR. The OHHL:LuxR complex dimerizes, binds to DNA, and promotes transcription of the lux operon, which produces bioluminescence. B) The structure of QscR (PDB 3SZT) bound to N-(3-oxo)-dodecanoyl L-homoserine lactone (OdDHL, shown as red spheres in the green monomer) with tryptophan residues shown as pink (weakly conserved) to purple (highly conserved) spheres. Inset: view of the positions of the tryptophan side chains (purple) in proximity to the ligand (red). C) A sequence alignment of a portion of several commonly studied LuxR-type receptors. Yellow highlights are highly conserved residues and purple highlights are the conserved and non-conserved tryptophan residues of QscR. D) The normalized absorption (dashed lines) and emission (solid lines) spectra for QscR, dansyl (dansyl amide; CAS 143139-6), and coumarin (7-hydroxycoumarinyl-4-acetic acid; CAS 6950-82-9). E) FRET occurs when the fluorophore-AHL conjugate is bound to the receptor; upon incubation with a competing ligand, there is no FRET signal. 
Several prominent Gram-negative bacterial pathogens, including Pseudomonas aeruginosa, utilize LuxI/LuxR-based QS to regulate a broad range of virulence phenotypes, including the production of toxins and growth into sessile biofilms. ${ }^{1,4}$ QS has attracted significant recent attention as a target to control bacterial infections in humans, and a number of chemical biology approaches have been aimed towards disrupting this communication process in order to attenuate bacterial virulence. ${ }^{4,5}$ Considerable research has focused on blocking the binding of the native AHL signal to its cognate LuxR-type receptor protein. Rational design, computational modeling, and high-throughput screening (HTS) using cell-based reporter assays have revealed small molecule modulators of several LuxR-type receptors. ${ }^{6-12}$ However, further research is needed to improve the potency, ${ }^{4,11}$ selectivity, ${ }^{13}$ and physicochemical properties ${ }^{14,15}$ of these chemical probes. Such development has been stymied by the limited mechanistic information of the modes by which they interact with and modulate the function of LuxR-type receptors, either as agonists or antagonists.

Quantitative direct-binding assays to characterize LuxR-type receptor-ligand binding could aid significantly in the maturation of chemical probes to study of QS; however, these methods, either in vitro or in-cell, are largely non-existent. Rather, small molecules that modulate LuxR-type receptor activity are typically characterized using either cell-based transcriptional reporter or phenotypic assays; both assay types offer limited information about the direct target of the small molecule, the affinity of the small molecule for its target, and the mechanism whereby the small molecule alters the activity of the target. While the potency $\left(E C_{50}\right)$ of a compound in a cell-based reporter assay is generally used as a proxy for affinity to the LuxR-type receptor, the direct relationship between reporter assay potency and ligand binding affinity has yet to be thoroughly examined in any LuxR-type receptor.

Two challenges have hindered the direct examination of LuxR-type receptor ligand binding: (1) inability to isolate the apo-receptor and (2) resistance of receptor:ligand complexes to ligand exchange. In the absence of a ligand, many LuxR-type receptors are insoluble, and as 
a result, LuxR-type receptors are typically overexpressed and purified in the presence of their native $A H L$ ligand. ${ }^{3,16-18}$ Additionally, while native $A H L$ disassociation and ligand exchange has been demonstrated for $\mathrm{LuxR}^{19}$ and $\mathrm{CViR}^{7}{ }^{7}$ several receptors resist ligand exchange in vitro when purified with their native ligand: TraR (Agrobacterium tumefaciens) ${ }^{16}$ LasR $\left(P\right.$. aeruginosa) ${ }^{20}$ and QscR ( $P$. aeruginosa) ${ }^{21}$ Direct characterization of ligand-binding affinity has been limited to a only few studies, including retention of tritium-labeled native AHL in cells expressing LuxR, ${ }^{19}$ isothermal calorimetry (ITC) in SdiA (E. coli) $)^{22}$ and CarR (Erwinia carotovora) (two receptors among the rare LuxR-type family members that are stable in their apo form), ${ }^{23}$ and changes in the fluorescence intensity of tryptophan residues in the AHL-binding site in apo-CarR as reported by Welch et al. ${ }^{24}$ These methods are either highly specialized or limited to certain receptors and have not been adopted as general approaches to examine ligand binding in LuxR-type proteins.

Fluorescence-based techniques represent some of the most sensitive, robust, and widely used assays for the study of ligand-receptor interactions. Albeit limited to CarR, Welch's tryptophan fluorescence quenching approach demonstrates their utility, yet relied on observing small differences in the fluorescence intensity of the apo-receptor and the receptor:ligand complex (or between two different receptor:ligand complexes). ${ }^{24}$ One strategy to bypass this complication and extend fluorescence based methods to a far wider array of LuxR-type receptors would be to utilize Förster Resonance Energy Transfer (FRET). ${ }^{25}$ Efficient energy transfer requires donor and acceptor fluorophores to be 10 to $100 \AA$ apart, and LuxR-type receptors conveniently have two highly conserved tryptophan residues in the ligand-binding site (e.g., W62 and W90 in QscR) and one in the helix-turn-helix domain (W186 in QscR) that could potentially donate energy to an AHL-fluorophore conjugate (Fig. 1B-C). ${ }^{3}$ Tryptophan can be excited at $280 \mathrm{~nm}$ and emits at around $330 \mathrm{~nm}$, which then can excite common fluorophores such as dansyl and coumarin (the large emission-excitation overlap shown in Fig. 1D). ${ }^{26}$ Such an experiment would measure the fluorescence of the receptor:FRET probe complex, rather 
than a mixture of receptor:ligand complexes, resulting in a robust signal that could be linearly related to the quantity of receptor:FRET probe complex. We hypothesized that this intrinsic FRET assay design could be applied to measure LuxR-type protein:ligand binding interactions quantitatively both in vitro and in-cell through competitive ligand displacement assays (as depicted in Fig. 1E).

In the current study, we report the construction of a set of synthetic AHL-fluorophore conjugates, the identification of six specific LuxR-type receptor-FRET probe pairs, and the application of this assay to gather the first quantitative binding data for non-natural AHLs with LuxR-type proteins. Our assay leverages the ability to produce LuxR-type proteins bound to weakly agonizing ligands and the subsequent displacement of these weak agonists with ligands of interest, a technique first demonstrated to be feasible for QscR by Oinuma and Greenberg ${ }^{21}$ yet unexplored further. We reasoned that this exchange approach could be applied broadly to facilitate ligand exchange for other LuxR-type receptors and thus enable the FRET assay. As proof-of-concept, we first established a robust in vitro FRET binding assay with purified QscR. Thereafter, we developed an analogous in-cell FRET binding assay that relies on soluble overexpression of QscR. We found that this in-cell binding assay could be readily applied for the study of five other LuxR-type receptors, and it should be readily adaptable to others. Lastly, we applied the FRET binding assays to address several basic questions concerning previously reported small molecule modulators of LuxR-type receptors: namely, do non-AHL ligands bind competitively with AHLs? Does iterative ligand development that relies on cell-based reporter data correlate with improvements in binding affinity? And, what is the relationship between ligand binding affinity and receptor stabilization? This FRET assay provides powerful new entry into advancing small molecule modulators of LuxR-type receptors as chemical tools and for delineating the fundamental molecular mechanisms by which both native and non-native small molecules alter receptor activity. 


\section{RESULTS}

\section{Fluorescent AHL probe design and synthesis.}

We designed a FRET-probe library that fused the common homoserine lactone (HSL, Fig. 1A inset) headgroup to a fluorophore with a variable linker length. We reasoned that compounds with 1-10 atom long linkers (DA0-6 and CU0-6; Fig. 2A) would mimic the acyl tail lengths found in most naturally occurring AHLs (i.e., 4-16 carbons), ${ }^{3}$ while compounds with the 14 and 15 atom linkers (DA11 and CU11; Fig. 2A) would likely be too large to reside in the enclosed ligand-binding pocket (assuming that these analogs target the AHL-binding site). Many nonnative $\mathrm{AHL}$ analogues with aryl and bicyclic tail substituents have been shown to be strongly active as LuxR-type modulators (as both agonists and antagonists; see examples in Fig. S1), ${ }^{6,12,27}$ suggesting that small fluorophores such as dansyl or coumarin could potentially be accommodated by LuxR-type receptors. We synthesized a small library of AHL-based FRETprobes in which HSL was linked to a dansyl or a 7-hydroxy coumarin fluorophore with aliphatic linkers of varying lengths via standard solution-phase coupling reactions (Fig. 2A). The spectral characteristics of each compound, including absorption maximum, extinction coefficient, fluorescence emission maximum, and quantum yield were determined, and in most cases, conjugation to the linker/HSL caused minimal perturbation to these characteristics relative to the parent fluorophore (Fig. S2-S5 and Table S2). 


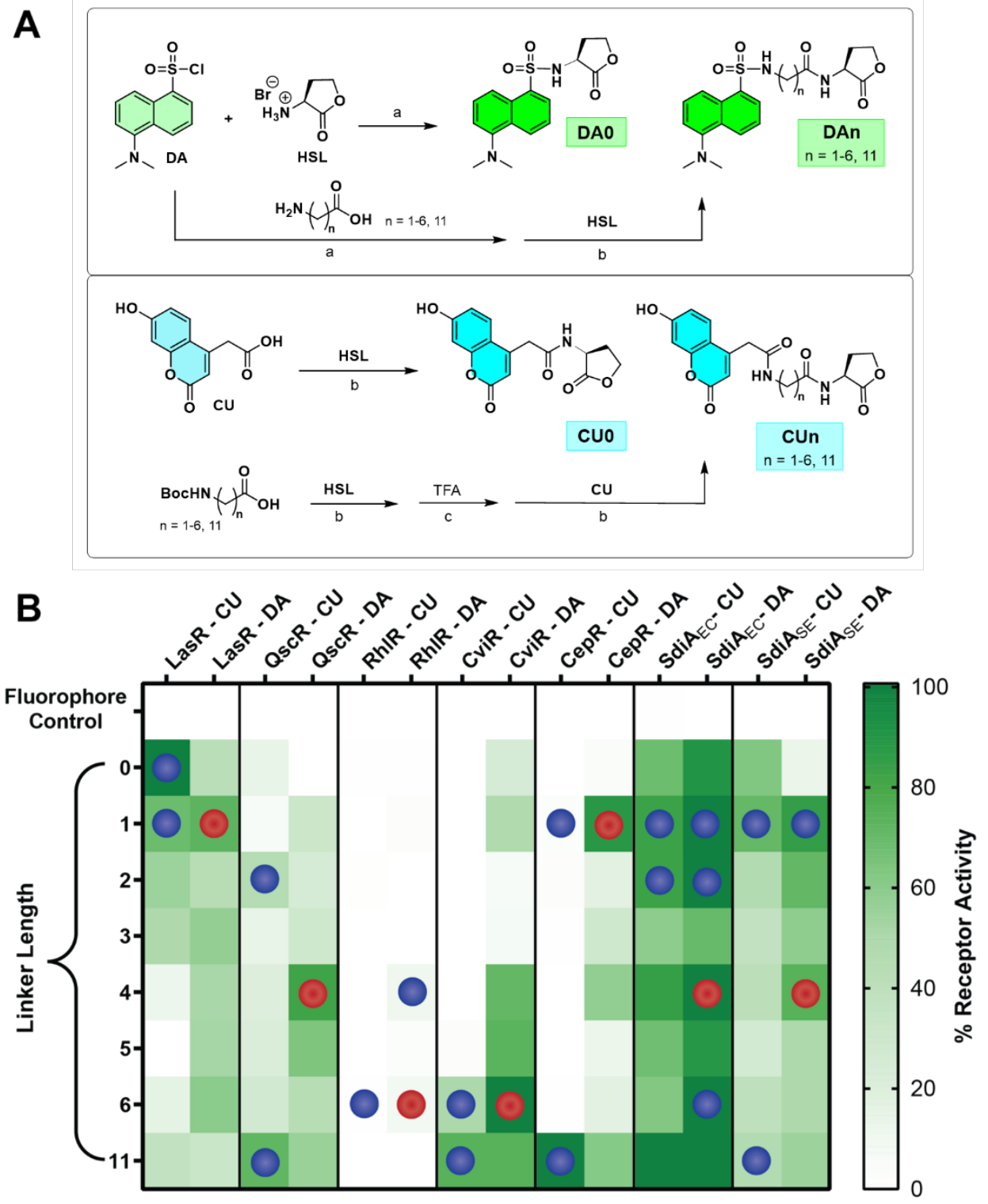

Figure 2. A) Synthesis and structures of AHL-based FRET-probe library. Reagents and conditions: a) sat. $\mathrm{NaHCO}_{3}$ (aq.), triethylamine (TEA) in acetone, $1 \mathrm{~h}$, room temperature (rt); b) N-(3-

Dimethylaminopropyl)- $N$ '-ethylcarbodiimide hydrochloride (EDC-HCl), 4-(dimethylamino)pyridine (DMAP) in $\mathrm{CH}_{2} \mathrm{Cl}_{2}$ (DCM), $16 \mathrm{~h}$, rt; c) 1:1 TFA:DCM, $1 \mathrm{~h}$, rt (TFA = trifluoroacetic acid). See Methods for details of compound characterization. B) LuxR-type receptor agonism assay data heatmap for the FRET-probe library. Each member of the FRET-probe library was assayed at $10 \mu \mathrm{M}$ for activity with each receptor listed on the top of the heatmap in an E. coli reporter strain (see Table S1 for strain details). Each receptor-FRET-probe pair has its own square in the heatmap. The heatmap is organized vertically by linker length, horizontally by receptor, and within a column by fluorophore (coumarin (CU) on the left and dansyl (DA) on the right). "Fluorophore control" refers to 7-hydroxy-4-acetic acid coumarin and dansyl amide for CU and DA, respectively. Receptor activity normalized for each strain as the activation of the reporter with saturating native ligand $(100 \%)$ and no ligand added $(0 \%)$. See Methods for native ligands and concentrations used for each positive control. Each square indicates an average activity of six technical replicates. Receptor:FRET-probe pairs selected for dose-response analysis indicated with blue or red spheres (curves in Fig. S7). Red spheres indicate receptor:FRET-probe pairs used in FRET assays (Fig. 5). 


\section{Receptor:FRET-probe pairs identified by reporter assays.}

We investigated the activity of the FRET-probes in various LuxR-type receptors using cell-based reporter assays. Because LuxR-type receptors are typically selectively activated by native AHLs with discrete acyl tail lengths, we reasoned that we would need to identify unique FRETprobe:receptor pairs as opposed to focusing on developing one universal FRET-probe. The FRET-probe library was screened for agonism (Fig. 2B) and competitive antagonism (against the native AHL; Fig. S6) in E. coli $\beta$-galactosidase reporter strains for the following seven LuxRtype receptors: LasR, ${ }^{6} \mathrm{RhIR},{ }^{28}$ and $\mathrm{QscR}{ }^{27}$ from $P$. aeruginosa; CviR from Chromobacterium violaceum; CepR from Burkholderia cenocepacia; ${ }^{29}$ SdiA from E. coli (referred to as SdiA $\left.\mathrm{AC}\right)^{12}$ and SdiA from Salmonella enterica (referred to as SdiA $\mathrm{SE}$ ). ${ }^{30}$ Efficacious agonists were identified in almost every receptor (Fig. 2B), with the coumarin derivatives generally less active than their dansyl counterparts. Interestingly, no efficacious antagonists were found (Fig. S6). At least three agonistic FRET-probes were selected for each receptor (as indicated in Fig. 2B by red and blue spheres) and further characterized via dose-response analyses (Fig. S7 and Table S3) in the reporter strains to determine their efficacy (maximum activation) and potency $\left(\mathrm{EC}_{50}\right)$. Apart from RhIR, we were able to identify FRET-probes that maximally activated each receptor with potencies ranging from $33 \mathrm{nM}$ to $32 \mu \mathrm{M}$ (probes selected for subsequent in-cell FRET assays indicated by red spheres in Fig. 2B).

As expected, we did not identify a single FRET-probe that agonized all seven LuxR-type receptors. In fact, we found that certain LuxR-type receptors with similar native AHL ligands displayed different selectivities for our FRET-probes. LasR and QscR both recognize the longtailed native $\mathrm{N}$-(3-oxo)-dodecanoyl-L-homoserine lactone (OdDHL) signal yet are maximally activated by different sized FRET-probes: the short linker probes (CU0 and DA1) for LasR and medium linker analogs (CU2 and DA4) for QscR. Likewise, CviR and CepR have similarly sized native ligands ( $N$-hexanoyl L-homoserine lactone $(\mathrm{HHL})$ and $\mathrm{N}$-octanoyl L-homoserine lactone 
$(\mathrm{OHL})$, respectively) but are maximally activated by differently sized FRET-probes: Iong chain CU6 and DA6 probes for CviR and the short chain DA1 probe for CepR. As observed in prior work demonstrating their promiscuity with regards to AHL-type ligands, ${ }^{12,30} \mathrm{SdiA} \mathrm{AC}_{\mathrm{E}}$ and SdiASE were strongly activated by most of the FRET-probes. RhIR showed little to no activation by any of the compounds in the probe library at $10 \mu \mathrm{M}$; the probe DA6, with $15 \%$ activation at $100 \mu \mathrm{M}$, was the most efficacious agonist of RhIR found. Unexpectedly, the very long linker probes, CU11 and DA11, were active in several of the receptors tested. Gomes et al. reported a similar finding in an earlier study, in which an AHL-fluorophore conjugate with a 16-atom linker between the HSL and a large Rhodamine B motif agonized CepR in a cell-based transcriptional reporter and was selectively retained in cells overexpressing CepR. ${ }^{31}$ Our results for CU11 and DA11 suggest (if they target the AHL-binding site) that either the binding pockets of these LuxR-type receptors are able to expand to fully accommodate these large ligands, or that their long tails are able to extend out of the binding pocket.

\section{QscR-DA4 in vitro FRET binding and displacement assays.}

We investigated whether the AHL-fluorophore probes could produce a FRET signal when bound to LuxR-type receptors and the application of this technique as an in vitro binding assay. For our initial studies, we selected QscR because this receptor is known to be amenable to in vitro ligand exchange when purified in the presence of a low-potency AHL agonist (specifically, $N$-(3oxo)-hexanoyl L-homoserine lactone (OHHL), as introduced above (see SI for methods). ${ }^{21,32}$ The FRET-probe DA4 was chosen for these initial studies because its $\mathrm{EC}_{50}$ in the cell-based reporter (133 nM, Table S3) was intermediate between that of OHHL (the purification ligand) and OdDHL (QscR's preferred native ligand), suggesting that DA4 could both displace any remaining bound $\mathrm{OHHL}$ and allow for ligand exchange in future displacement assays (depicted in Fig. 3A). 
First, we examined whether QscR-DA4 complexes produced a FRET signal. We introduced DA4 to the purified QscR:OHHL complex, incubated for $30 \mathrm{~min}$ at room temperature, excited at $280 \mathrm{nM}$, and measured a strong emission signal at $530 \mathrm{~nm}$ (Fig. 3B). To determine whether this excitation signal was specific to QscR-DA4 binding, we measured fluorescence for several control samples: QscR:OHHL plus dansyl amide (DNSA), the parent fluorophore which lacks the HSL head group and is inactive in QscR (Fig. 2); DA4 plus bovine serum albumin (BSA) as a non-specific protein control; and QscR:OHHL plus DA4 in the presence of competing native ligand (OdDHL) at high concentration. Consistent with specific QscR-DA4 binding, no appreciable fluorescence emission was observed in these control experiments (Fig. 3B). The result of the latter control experiment with OdDHL supports DA4 binding in the AHLbinding site. We note that QscR has five tryptophans that could serve as the FRET donor(s), three of which are highly conserved and have been shown to be important to LuxR-type receptor activity; ${ }^{33}$ therefore, we could not examine the activity of a QscR mutant lacking tryptophans as a control for the origins of the FRET signal. Nevertheless, the results of these experiments are consistent with an intrinsic FRET event occurring between DA4 and the QscR receptor. 


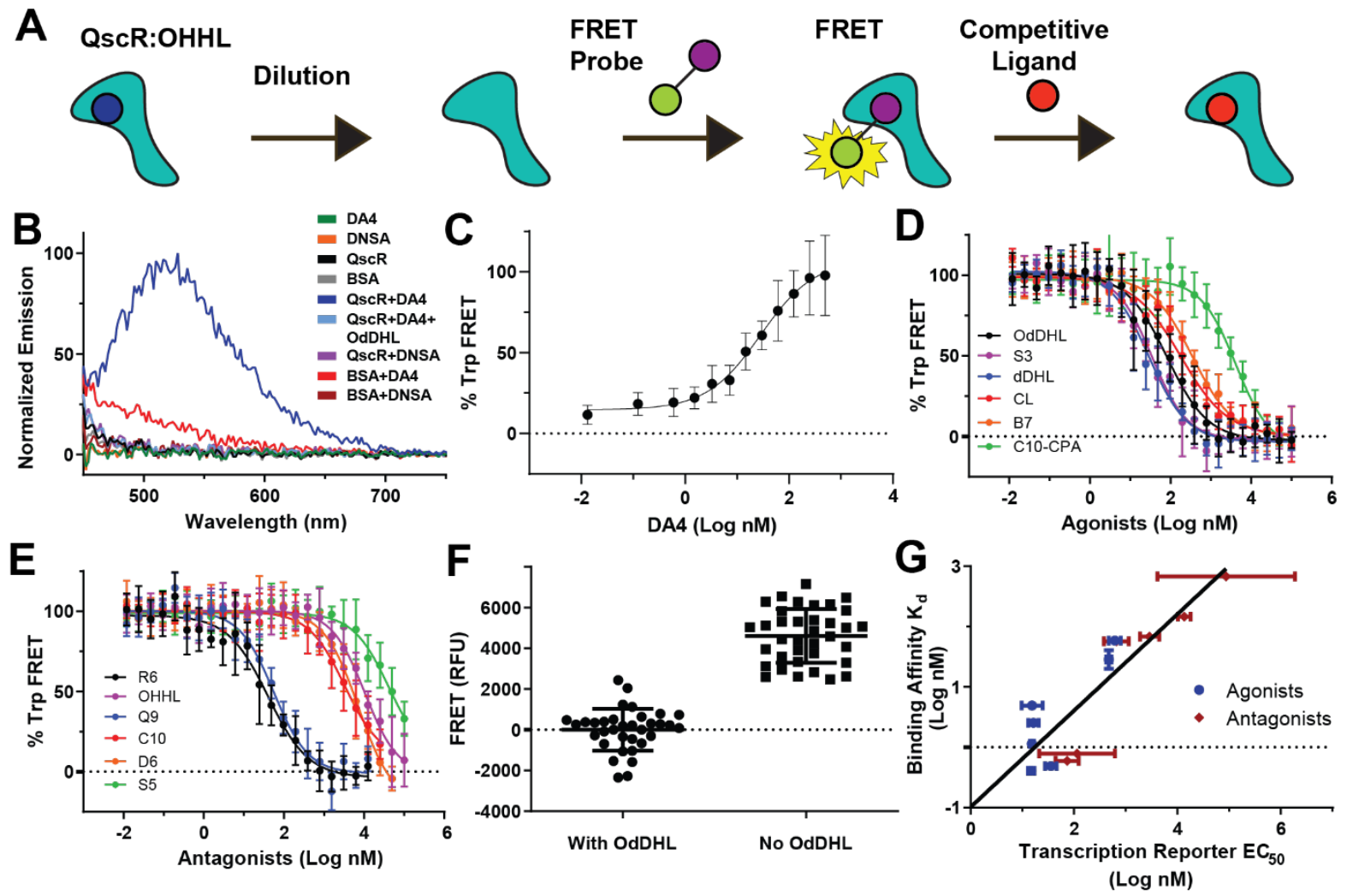

Figure 3. A) Schematic of the in vitro FRET-probe binding and ligand displacement approach. Purified QscR bound to a weak agonist (OHHL) is diluted in step 1 to promote disassociation, followed by binding of a FRET-probe in step 2, and then displacement with a ligand of interest in step 3. B) Fluorescence emission spectra (ex $280 \mathrm{~nm}$ ) of the QscR-DA4 FRET pair and control samples. Each data is the average of technical duplicates and normalized to the fluorescence intensity of QscR+DA4. All components (fluorophore: DA4 or dansyl amide control (DNSA); protein, QscR:OHHL or bovine serum albumin control (BSA); and OdDHL were held at $1 \mu \mathrm{M}$. C) Dose response curve for DA4 binding to QscR (2 nM). Each concentration was measured as three independent replicates, each with technical triplicates. Dose response curves of D) synthetic agonists and E) synthetic antagonists displacing DA4 $(2 \mu \mathrm{M})$ from QscR $(5 \mathrm{nM})$. Each concentration was measured as three independent replicates, each with five technical replicates. F) Replicates for Z-factor analysis for the in vitro FRET DA4 displacement assay in QscR. QscR $(5 \mathrm{nM})$ with DA4 $(2 \mu \mathrm{M})$ was treated with OdDHL $(50 \mu \mathrm{M})$ or with vehicle control $(\mathrm{n}=36)$. G) Correlation between potencies determined from cell-based transcriptional reporters $\left(E_{50}\right)$ and affinities $\left(\mathrm{K}_{\mathrm{d}}\right)$ determined using the in vitro FRET displacement assay for a set of QscR agonists (in blue) and antagonists (in red). All the antagonists of QscR display partial agonism and this partial agonism was used to determine the $\mathrm{EC}_{50}$ plotted here. See Table S4 for further details and Methods for description of data handling.

We next determined the affinity of the QscR-DA4 interaction by measuring FRET with varying concentrations of DA4 (Fig. 3C). We designed this experiment such that we could calculate a binding affinity $\left(\mathrm{K}_{\mathrm{d}}\right)$ from standard saturation binding equations (see Methods), 
including determining the appropriate incubation period for equilibrium measurements and using a sufficiently low concentration of QscR to avoid ligand depletion (see SI Note 1 for details). ${ }^{34,35}$ We calculated a $K_{d}$ of $28 \pm 10$ nM for DA4 using the FRET assay; the Hill slope for the curve in Figure $3 C$ is not statistically different than $1.0(p=0.05)$, indicating that DA4 binds tightly to a single site on QscR (which is monomeric under these conditions). ${ }^{21}$ This value was $\sim 5$-fold lower than its $\mathrm{EC}_{50}$ measured in the cell-based reporter assay of QscR activity (133 nM, Table S3). This result suggests that either the in-cell affinity or in-cell availability of DA4 is lower than in vitro.

We sought to explore whether displacement of DA4 from QscR could be utilized as a general assay to determine the binding affinity of other ligands. We selected a set of known QscR modulators for these displacement assay that was comprised of six agonists and six antagonists (structures shown in Fig. S8). ${ }^{27}$ In cell-based reporter assays, all of the agonists strongly activate QscR (>85\% max activation), and all of the antagonists are able to reduce the activity of QscR in competition against OdDHL (between 35 and 94\% max inhibition) (Figure S9 and Table S4). We note that these antagonists actually display partial QscR agonism, that is, they are able to agonize QscR but not fully; small molecule QscR antagonists that lack this activity profile have not been reported..$^{32}$ For the in vitro FRET ligand displacement experiments, we added varying concentrations of competing ligand to a solution of $5 \mathrm{nM}$ QscR and $2 \mu \mathrm{M}$ DA4 and measured FRET. The competition displacement dose-response curves are shown in Figures 3D (agonists) and 3E (antagonists). We calculated OdDHL to have an $\mathrm{IC}_{50}$ value of 82 $\mathrm{nM}$ in the displacement assay (Fig. 3D) and a binding affinity $\left(\mathrm{K}_{\mathrm{d}}\right)$ of $1.3 \pm 0.4 \mathrm{nM}$ when converted using the Cheng-Prussoff equation (see Methods). This affinity is comparable to the 3.1 nM affinity for QscR:OdDHL reported by Oinuma and Greenberg as estimated from a DNA shift assay. ${ }^{21}$ The $K_{d}$ values of each of the remaining five agonists and the six antagonists were calculated similarly from the $\mathrm{IC}_{50}$ values from the curves in Figure 3D-E, giving values that ranged from $0.4 \mathrm{nM}$ to $681 \mathrm{nM}$ (Table S4). The Z-factor for this displacement assay was 
calculated to be 0.59 (data used in this calculation shown in Fig. 3F), suggesting that this assay would perform well in a high throughput screening format. ${ }^{36}$ The results of these experiments support this FRET-based displacement assay as a straightforward and generalizable method to measure QscR:ligand binding in vitro.

We wanted to further investigate the relationship between the potency of a ligand in a cell-based reporter assay and the affinity of a ligand in vitro in the FRET displacement assay. Comparison of the $\mathrm{EC}_{50}$ values for the QscR agonists and antagonists in the reporter assay to their $K_{d}$ values for QscR in the FRET assay (Fig. 3G) using a Pearson Correlation (see Methods for details) showed they were highly correlated (Pearson Coefficient $r=0.9$ ), indicating that the cell-based transcription reporter potency, at least for QscR, is a generally accurate proxy for receptor affinity. However, two important deviations were identified. Firstly, the antagonists were less potent in reporter assays than their affinities would predict; specifically, compounds R6 and Q9 bind slightly tighter to QscR than OdDHL but have $\mathrm{EC}_{50}$ values 5-7 times higher in cellbased reporter assays. Congruent with the latter observation, these two QscR-antagonist complexes have been reported to bind DNA, but with a much lower affinity than QscR-OdDHL. ${ }^{32}$ Secondly, the four highly potent agonists (OdDHL, dDHL, CL, and B7) have approximately the same $\mathrm{EC}_{50}$ value $(15 \mathrm{nM})$ in the cell-based reporter assays, but their binding affinities span more than an order of magnitude $(0.4-4.9 \mathrm{nM})$, indicating that the reporter assay is unable to report accurately on the affinities of highly potent ligands. These two findings highlight the limitations of cell-based reporter assays to accurately convey differences in affinity when developing highly potent agonists and antagonists.

\section{QscR-DA4 in-cell FRET binding and displacement assays.}

Due to the challenges of isolating most LuxR-type receptors in vitro, even in the presence of an agonist, ${ }^{10,32,37}$ we recognized that an in-cell direct binding assay could be valuable. To this end, we investigated the feasibility of performing the DA4 displacement assay in E. coli 
overexpressing QscR. For this assay, we overexpressed QscR in the presence of OHHL under the same conditions used for protein purification (see Methods). The E. coli cells were washed to remove any unbound $\mathrm{OHHL}$, diluted to a low cell density, and incubated with DA4 for the binding assay or DA4 plus a competitive ligand for competition assays (shown schematically in Fig. 4A). Because of the complexity of the cellular environment in comparison to in vitro conditions, we needed a means of separating FRET signal arising from DA4:QscR interactions and FRET signal arising from potential interactions of DA4 with other cellular components. To do so, the FRET signal of cells not producing QscR was subtracted from the FRET signal of induced cells to remove FRET signal arising from non-specific binding interactions. 


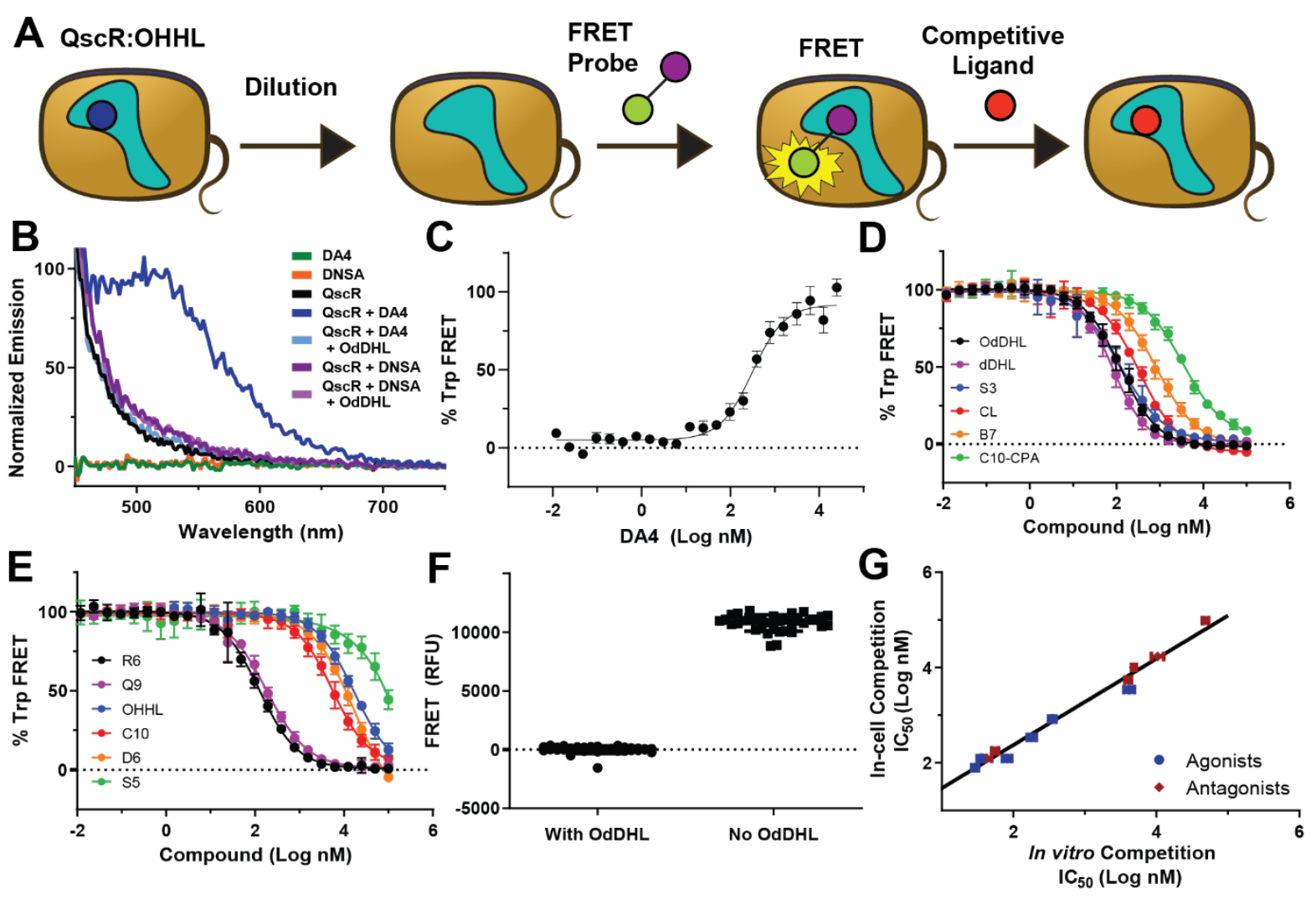

Figure 4. A) Schematic of the in-cell FRET-probe binding and ligand displacement approach. E. coli overexpressing QscR in the presence of a weak agonist are diluted in step 1 to promote disassociation, followed by binding of a FRET-probe in step 2, and then displacement with a ligand of interest in step 3. B) Fluorescence emission spectra (ex $280 \mathrm{~nm}$ ) of the QscR-DA4 FRET pair and control samples in $E$. coli. All compounds (DA4, dansyl amide control (DNSA), and OdDHL) held at $1 \mu \mathrm{M}$. Overexpressed cells diluted to OD600 of 0.05 . Each data set is the average of technical duplicates. C) Dose response of DA4 binding to QscR in E. coli. Each concentration was measured as four independent replicates, each with five technical replicates. Dose response curves of synthetic $\mathbf{D}$ ) agonists and $\mathbf{E}$ ) antagonists displacing DA4 $(2 \mu \mathrm{M})$ from QscR in E. coli. Each concentration was measured as three independent replicates, each with technical triplicates. F) Replicates for Z-factor analysis for in-cell FRET DA4 displacement from QscR. QscR (5 nM) with DA4 $(2 \mu \mathrm{M})$ was treated with OdDHL $(50 \mu \mathrm{M})$ or vehicle control $(\mathrm{n}=36)$. G) Correlation between the $\mathrm{IC}_{50}$ values for displacement of $2 \mu \mathrm{M}$ DA4 by QscR agonists (in blue) and antagonists (in red) as measured by FRET in vitro and in-cell. See Table S4 for further details and Methods for description of data handling.

We developed and characterized the in-cell FRET assay protocol in the same manner as the in vitro FRET assay for QscR described above. We measured the FRET signal between DA4 and the QscR:OHHL complex produced in-cell and observed a strong FRET emission at $530 \mathrm{~nm}$ upon addition of DA4 (Fig. 4B). Like the in vitro assay, this emission was specific to the presence of DA4 and eliminated by the addition OdDHL, suggestive of competitive ligand 
displacement. We obtained a well-behaved ligand binding curve by varying the amount of DA4 in the presence of dilute E. coli producing QscR:OHHL (Fig. 4C). However, we did not assign a $\mathrm{K}_{d}$ value using this assay as we could not accurately determine the concentration of QscR or the amount of "free" DA4 in cells; therefore, we calculated an $\mathrm{EC}_{50}$ value from the dose response curve for D4A (which we term an apparent affinity here) that is dependent on these specific sample preparation conditions. We observed an 11-fold decrease in apparent affinity as compared to the in vitro $\mathrm{K}_{d}$ measurement above ( $313 \mathrm{nM}$ in Fig. $4 \mathrm{C}$ vs. $28 \mathrm{nM}$ in Fig. $3 \mathrm{C}$ ); this discrepancy could reflect our inability to accurately determine the amount of "free" DA4 due to its level of non-specific binding or potential partitioning into the cell membrane (DA4 has a LogP of 2.76).

We next applied the in-cell FRET assay to measure ligand displacement by agonists (Fig. 4D) and antagonists (Fig. 4E), analogous to the in vitro FRET assay above. These in-cell assays displayed remarkably high signal-to-noise ratios, allowing for robust model fits. The Zfactor for this displacement assay was 0.72 , indicating that this assay, like the in vitro method, would be readily applicable in high-throughput screening (Fig. 4F). OdDHL was found to have an $\mathrm{IC}_{50}$ in-cell very similar to the in vitro displacement assay (124 nM in-cell vs. $82 \mathrm{nM}$ in vitro), and a comparison of the $\mathrm{IC}_{50}$ values for all the QscR agonists and antagonists obtained in vitro vs. in-cell is shown in Figure 4G. The tight correlation (Pearson Coefficient $r=0.99$ ) between these data suggests that this in-cell FRET assay can accurately capture differences in in vitro ligand-binding affinity between compounds across a wide range of affinities for QscR. Again, these differences are not accurately conveyed for potent QscR agonists and antagonists by cellbased transcriptional reporter assays.

\section{In-cell FRET assays with other LuxR-type receptors.}

We evaluated if this in-cell FRET assay could be applied to other LuxR-type receptors beyond QscR. First, we needed to select the appropriate receptor:FRET-probe pairs and determine 
overexpression conditions that produced soluble receptor with a ligand that would readily disassociate. We focused in on three dansyl FRET-probes that were active in the cell-based reporter strains examined above (DA1 in LasR and CepR; DA4 in SdiA $A_{E C}$, and SdiAsE; and DA6 in RhIR and CviR; see red spheres in Fig. 2B); each of these probes displayed an intermediate potency in cell-based reporter assays, suggestive that they could be displaced by competitive ligand ( $\mathrm{EC}_{50}$ values of 2.6 to $32 \mu \mathrm{M}$; the $\mathrm{EC}_{50}$ of DA6 for RhIR not calculated due to solubility constraints). When optimizing receptor overexpression conditions, we sought conditions that both solubilized the receptor and allowed for ligand exchange. Ligand exchange experiments have been reported for CviR purified with its native ligand, $\mathrm{HHL},{ }^{7}$ and Sdi $\mathrm{A}_{E C}$ is soluble in the absence of ligand; ${ }^{22}$ therefore, $\mathrm{HHL}$ or no $\mathrm{AHL}$ was used for CviR and SdiA $\mathrm{EC}_{\mathrm{EC}}$ production, respectively. LasR has been reported to be solubilized by both its native ligand OdDHL and the much less potent $\mathrm{N}$-(3-oxo)-octanoyl L-homoserine lactone (OOHL; $\mathrm{EC}_{50}=4.5 \mu \mathrm{M}$ vs. $2 \mathrm{nM}$ for OdDHL in cell-based reporter assays); ${ }^{17,38}$ we selected both of these AHLs to test the FRET assay with LasR. Ligand exchange experiments, let alone protein production experiments, remain largely unreported for RhIR, ${ }^{10} \mathrm{CepR}$, and SdiAsE. Thus, for these proof-of-concept studies, we simply sought to identify ligands that allowed for solubilization of these receptors when produced in E. coli. RhIR is not well solubilized by its native ligand, $\mathrm{N}$-butanoyl Lhomoserine lactone (BHL; $\mathrm{EC}_{50}=9 \mu \mathrm{M}$ in the cell-based reporter assay), but we found that the non-native AHL S4 $\left(\mathrm{EC}_{50}=1.6 \mu \mathrm{M}\right)^{39}$ was able to solubilize RhIR (Fig. S10). In turn, CepR was solubilized by its native ligand, octanoyl L-homoserine lactone $\left(\mathrm{OHL} ; \mathrm{EC}_{50}=6 \mathrm{nM}\right)$, and SdiAsE could be solubilized by OOHL $\left(\mathrm{EC}_{50}=1 \mathrm{nM}\right)$.

When overexpressed with the ligands discussed above, each receptor (except for CepR) produced a robust FRET signal in the presence of its paired probe, and this signal was abolished upon addition of a competing ligand (Fig. 5A) with signal-to-noise ratios $(\mathrm{S} / \mathrm{N})$ ranging from 12 to 177 . These results are consistent with the fluorophore probes displacing the 
overexpression ligands, intrinsic FRET occurring between the receptors and probes, and the subsequent displacement of the probes with a different ligand. In addition, these data indicate that under these assay conditions, for at least certain receptors (CviR, LasR, RhIR, and SdiAsE), the FRET probes could displace ligands with strong agonistic activity (i.e., with up to 10,000-fold lower $\mathrm{EC}_{50}$ values in cell-based reporter assays relative to the FRET probe). CepR produced with OHL, however, did not have a FRET signal significantly different than background, and we asked whether production of CepR with an agonist with reduced potency would permit more facile ligand exchange. We found that the weaker agonist $N$-decanoyl L-homoserine lactone $\left(\mathrm{DHL} ; \mathrm{EC}_{50}=110 \mathrm{nM}\right)$ could solubilize CepR, yet CepR did not yield a greatly improved FRET signal when overexpressed with $\mathrm{DHL}(\mathrm{S} / \mathrm{N}$ of 2.6) relative to $\mathrm{OHL}$. Potentially, this low $\mathrm{S} / \mathrm{N}$ for CepR stems from either the low potency of DA1 with CepR $\left(\mathrm{EC}_{50}=32 \mu \mathrm{M}\right.$ in cell-based reporter assays) or because DHL is still sufficiently potent to hinder ligand exchange. Interestingly, and in contrast to CepR, the LasR FRET signal was highly dependent on the choice of solubilizing ligand, with a 13-fold lower FRET signal observed for LasR when solubilized by the tightly bound OdDHL compared to the weakly bound OOHL (Fig. 5B). 


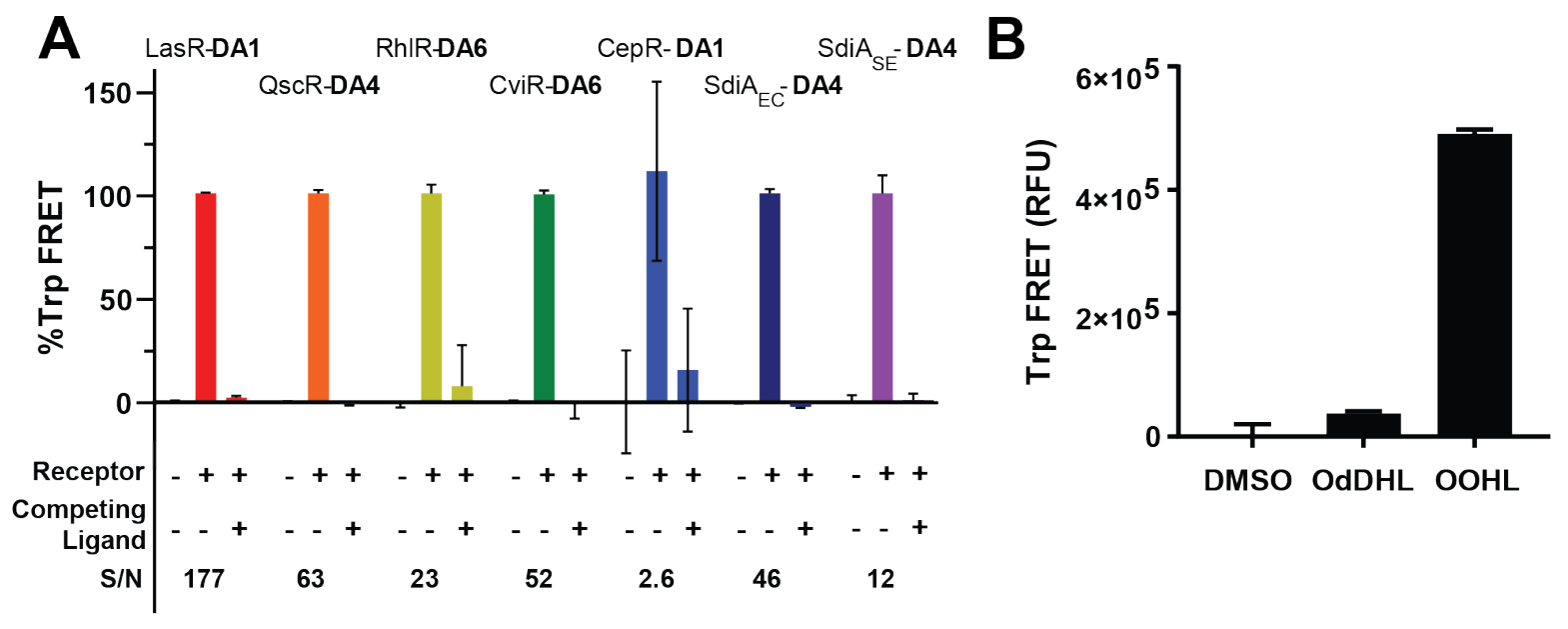

Figure 5. FRET assay data with receptors beyond QscR. A) In-cell FRET assay data for various LuxRtype receptor:fluorophore probe pairs (indicated at top of plot). The fluorophore was added to $E$. coli without overexpression of the receptor (-) or with overexpression of the receptor $(+)$ and without a competing ligand (-) or with $100 \mu \mathrm{M}$ of competing ligand (+) (indicated below the plot). DA1 used at 25 $\mu \mathrm{M}$, DA4 at $10 \mu \mathrm{M}$, and DA6 at $10 \mu \mathrm{M}$ for CviR and $50 \mu \mathrm{M}$ for RhIR. Overexpression ligands were OOHL, $\mathrm{OHHL}, \mathrm{S} 4, \mathrm{HHL}, \mathrm{DHL}$, none, and OOHL for LasR $\rightarrow$ SdiAsE (left $\rightarrow$ right at top of plot), respectively. Similarly, the competing ligands were OdDHL, OdDHL, S4, HHL, OHL, OOHL, and OOHL. See Methods for overexpression details and data handling. Each condition is the average of 8 or 16 replicates and error bars indicate standard deviation (SD). The signal-to-noise $(\mathrm{S} / \mathrm{N})$ ratio for each receptor-FRET pair is listed at the bottom of the plot. S/N calculated as the mean/SD of the FRET signal. B) The FRET signal from LasR when overexpressed without ligand (DMSO) or with $25 \mu \mathrm{M}$ OdDHL or OHHL and incubated with 25 $\mu \mathrm{M}$ DA1. Each condition has 16 replicates and error bars indicate SD.

We followed up these single concentration FRET experiments in LasR, SdiA $A_{E C}, \operatorname{Sdi} A_{S E}$, RhIR, and CviR with full dose-response characterization of (i) FRET-probe binding (analogous to Fig. 4C, see Table S5 and Fig. S11) and (ii) displacement by a competing ligand (analogous to Fig. 4D, see Fig. S12). While in certain cases the FRET-probe affinity was too low to achieve a plateau in FRET-probe binding assays, the observed FRET signal still permitted productive ligand displacement assays that allowed for comparisons in affinity between competitive ligands. Collectively, these assay data allow us to draw the following conclusions: first, this incell FRET assay is compatible with a range of LuxR-type receptors to monitor ligand binding, and second, using a lower potency ligand for solubilizing the LuxR-type receptor can greatly improve FRET signal but is unnecessary in some cases. The latter result has implications for biochemical and biophysical investigation of LuxR-type receptors beyond the FRET assays 
described here, as it provides a pathway for the manipulation and study of this often recalcitrant protein class in vitro.

\section{FRET assays to interrogate LuxR-type protein mechanism.}

The FRET assays described here should allow for many questions regarding LuxR-type protein activity to be addressed. For example, investigations of whether non-AHL derived small molecule QS modulators directly bind LuxR-type receptors or bind competitively in the AHLbinding pocket have rarely been performed. Indole has long been considered a potential chemical signal in bacteria and is produced by certain Gram-negative bacteria (reviewed recently by Zarkan et al.). ${ }^{40}$ Conflicting reports exist about whether indole directly interacts with and alters the activity of LuxR-type receptors. Indole has been reported to modulate QS in several species, including E. coli (via SdiA $\left.E_{E C}\right),{ }^{41} P$. aeruginosa (via either the las or $r h /$ system), ${ }^{42}$ and C. violaceum, P. chlororaphis, and Serratia marcescens (LuxR-type receptor targets unknown). ${ }^{43}$ Conversely, indole also has been reported to have no effect on SdiA $A_{E C}$ and $\mathrm{SdiA}_{\mathrm{SE}},{ }^{44}$ and it has been reported to only modulate $\operatorname{TraR}(A$. tumefaciens) and AqsR (Acinetobacter oleivorans) via a general effect on protein folding. ${ }^{45}$ In our own hands, we found that indole can activate SdiA $\mathrm{AC}_{\mathrm{C}}$ in a cell-based reporter assay (Fig. S13). To ask whether this compound can bind directly to SdiA $\mathrm{EC}_{\mathrm{C}}$ and other LuxR-type receptors, we tested indole in our incell FRET displacement assays for SdiA $A_{E C}, S_{d i} A_{S E}, C v i R$, and QscR, and observed no decrease in FRET, indicating that indole does not bind in the AHL binding pocket of at least these four receptors (Fig. 6A). These results support a non-competitive mechanism of action for indole if it targets these receptors. Similarly, we investigated three small molecules previously reported to strongly modulate LasR (in a range of cell-based and phenotypic assays) in the in-cell FRET displacement assay. ${ }^{38}$ These compounds were: brominated furanone C- $30^{46}$ and PD-12, ${ }^{47}$ both of which are debated to directly interact with LasR; V-06-18, which was identified as a lead LasR antagonist ${ }^{38}$ and the focus of a recent structure-activity relationship study; ${ }^{11}$ and the strong 
LasR agonist TP-1, which has been co-crystalized with the LasR ligand binding domain and binds in the OdDHL pocket. ${ }^{37,48}$ We found that C-30 and PD-12 are indeed able to partially displace DA1, albeit at very high concentrations (1 mM), V-06-018 displaces DA1 with an intermediate affinity, and TP-1, as expected, was able to displace DA1 from LasR with a similar affinity to its native ligand, OdDHL (Fig. 6B). These results underscore the utility of this in-cell assay technique for delineating the targets of QS modulatory ligands and obtaining quantitative data regarding their relative binding affinities.
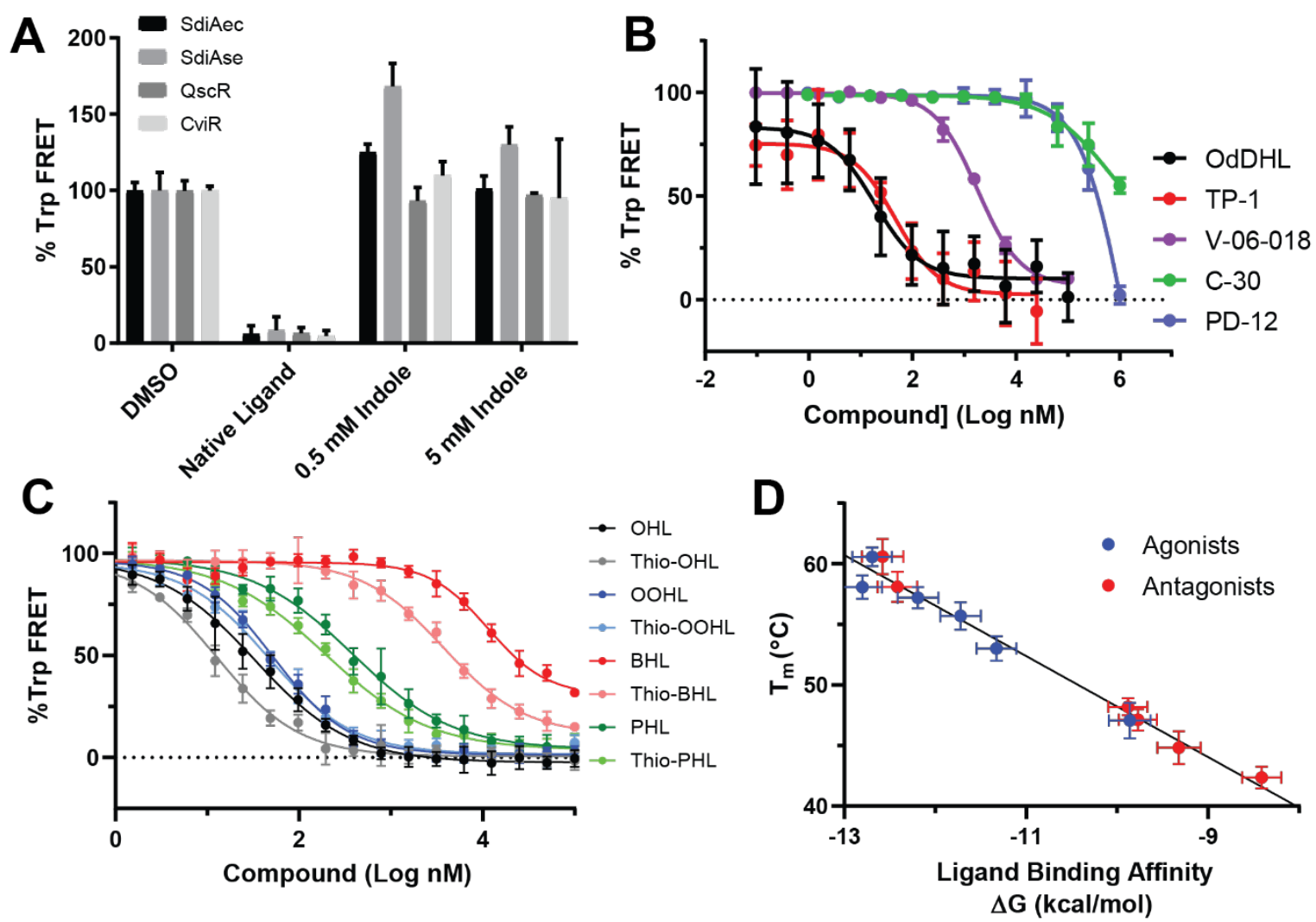

Figure 6. Mechanistic FRET assays with various small molecules. A) E. coli overexpressing the receptor listed were incubated with $1 \mu \mathrm{M}$ DA4 (DA6 for CviR) and either DMSO, $10 \mu \mathrm{M}$ native AHL signal, or indole (at the low and high estimates of indole production in E. coli). \% Trp FRET defined with $100 \%$ equal to the DMSO condition. Each condition is the average 8 replicates with the error bars indicating the SD. B) LasR was overexpressed and incubated with DA1 and varying concentrations of OdDHL, TP-1, V06-018, C-30, or PD-12. Each condition is the average 8 replicates with the error bars indicating the SD. C) In vitro DA4-SdiA $A_{E C}$ displacement FRET assay with AHL-thiolactone pairs. Error bars indicate SD of five technical replicates. D) Correlation between ligand binding affinity to QscR and the thermal stability of the QscR-ligand complex with agonists in blue and antagonists in red. See Figure S14 and Table S6 for further details. See Methods for details of assays and data handling. 
Structure-activity relationships for the known chemical probes of LuxR-type proteins have been typically determined using cell-based transcription reporter assays, which have the caveats outlined above. The FRET assay has the potential to determine whether a change in potency in these reporter assays reflects a change in affinity, allowing for more educated design and tailoring of ligands. As an example, we explored the activity of a set of thiolactone AHL analogs that have been explored as QS modulators ${ }^{14}$ and reported to have increased potency relative to their parent lactone analogs in cell-based reporter assays of several LuxR-type receptors - i.e., in SdiA $\mathrm{SE}^{30} \mathrm{SdiA}_{\mathrm{EC}},{ }^{12} \mathrm{RhIR},{ }^{15}$ and LasR. ${ }^{10}$ One explanation, aside from their increased hydrolytic stability over the time course of the assay, would be increased ligand affinity. We performed in vitro DA4 FRET displacement assays for several lactone-thiolactone pairs with purified SdiA $A_{E C}$ (see Methods for purification details) and found that SdiA $A_{E C}$ binds more tightly to the thiolactone analogues (Fig. 6C). The improvement in affinities for the thiolactones varies from 1.1-fold to 3.1-fold, suggesting that this particular modification not only can improve ligand stability but also affinity. ${ }^{12}$

Lastly, we applied the FRET assay to examine the stability of LuxR-type receptors. Despite the critical importance of ligand binding for LuxR-type receptor solubilization and activation (vide supra), only a few studies have directly characterized the ability of a ligand to increase the thermal stability of LuxR-type receptors. ${ }^{22,32,37}$ We reasoned that the in vitro FRET assay could allow us to directly investigate the relationship between ligand-binding affinity and LuxR-type receptor stability. First, we characterized the stability of QscR complexed to a set of QscR agonists and antagonists (used in Fig. 3D-E) via differential scanning fluorimetry (DSF) thermal shift assays, which provided the temperature at which QscR-ligand complexes unfold (see Methods and Fig. S14 for representative DSF data). We then compared these melting temperatures to the ligand affinity values as determined by in vitro FRET. As shown in Figure $6 \mathrm{D}$, the affinity of a ligand for QscR is highly correlated with the thermal stability of the QscRligand complex (Pearson Coefficient, $r=0.99$ ). Interestingly, this relationship holds for QscR for 
both agonists and antagonists (again, the latter being partial agonists; see above). These data are congruent with the hypothesis that the affinity of the ligand for QscR plays a large role in its thermal stability, as varying the affinity of the ligand from 0.4 to $681 \mathrm{nM}$ varies the $\mathrm{T}_{\mathrm{m}}$ by $18{ }^{\circ} \mathrm{C}$. Together, the three examples outlined here serve to demonstrate how this FRET assay can be applied to integrate our understanding of ligand binding into our broader understanding of LuxRtype receptor mechanism and QS signaling in general.

\section{DISCUSSION}

We report the development of a FRET assay that utilizes conserved endogenous tryptophans located in the LuxR-type receptor ligand-binding pocket and novel fluorophore-AHL conjugates as the fluorescence donor and acceptor, respectively. We synthesized a small library of fluorophore-HSL conjugates to mimic the varying sizes of naturally occurring AHLs. This library was screened against seven LuxR-type receptors to identify probes capable of agonizing each receptor in cell-based reporter assays. We then performed proof-of-concept experiments on one receptor-QscR from $P$. aeruginosa - to demonstrate the feasibility of this FRET assay system. We found that FRET could be used to measure differences in ligand binding affinity in vitro with purified QscR and in-cell using an E. coli strain overexpressing QscR. The in-cell method could be readily extended to other LuxR-type receptors, and we demonstrate its compatibility in five additional LuxR-type receptors using a discrete set of FRET probes. These latter experiments were enabled by the development of robust expression and solubilization techniques for each receptor complexed to a specific agonist ligand (if needed) that could be readily displaced by the FRET. Finally, we show that these binding assays can be used to address several basic questions concerning the targets and relative activities of known LuxR-type receptor/QS modulators and allow for the relationship between ligand binding affinity and receptor stability to be directly addressed for the first time. This assay represents a significant improvement in our ability to quantitatively measure ligand binding in vitro and in-cell for LuxR-type receptors. 
Given the simplicity of the probe synthesis and assay design, these FRET assays have the potential for widespread adoption by researchers studying LuxR-type receptors and their role in QS biology. On specific application where these techniques could find immediate utility is in the study of orphan or 'solo' LuxR-type receptors, an area that has exploded over the past decade. ${ }^{49}$ This class of LuxR-type receptors are found in species that do not produce AHLs, but rather sense AHLs (or other small molecules) produced by other bacteria (or other organisms) in their environment. ${ }^{49}$ Orphan receptors likely make up the majority of LuxR-type receptors ${ }^{50}$ yet face a significant barrier to study: namely, if we do not know what ligands activate an orphan LuxR-type receptor, then we cannot identify the genes regulated by the receptor. And in turn, if we do not know which genes are regulated by the receptor, then we cannot use transcriptional reporter assays to identify compounds that activate the receptor. This FRET assay provides a direct means to solve this chicken-or-the-egg problem; the FRET assay can be used to identify ligands that bind to an orphan receptor as a starting point for transcriptomics to identify genes that may regulate by the receptor.

The FRET assays will also facilitate the optimization of the current set of known QS modulators, the characterization of new or putative QS signals in general, and the examination of the biophysical properties of LuxR-type receptors. The assays are readily amenable to the large-scale screening of synthetic small molecule libraries or could be used in a fragment-based approach to developing competitive inhibitors of LuxR-type receptors. Of specific value in this context, the FRET assay allows for unambiguous determination of the effect on potency of a given structural modification, which could aid in the rational design of chemical tools for modulating QS with improved physicochemical properties. Finally, turning to the LuxR-type receptor target, the FRET assay should allow for quantitative determinations of changes in ligand-binding affinity upon alteration of the receptor (e.g., generated via point mutations or truncations, or when examined as monomers, dimers, or DNA bound complexes, etc.) that have thus far only been discussed largely qualitatively. For example, such experiments could readily 
and unambiguously address the question of whether these proteins bind their ligands cooperatively or noncooperatively. We are optimistic that these probes, assays, and the insights that they provide will advance the QS field.

\section{METHODS}

All methods are provided in the Supporting Information.

\section{SUPPORTING INFORMATION}

Supporting information available: Methods, additional data, and supplementary discussions. This material is available free of charge via the Internet.

\section{ACKNOWLEDGMENTS}

Financial support for this work was provided by the NIH (R35 GM131817). M.J.S. was supported in part by the UW-Madison NIH Biotechnology Training Program (T32 GM008349) and an NSF Graduate Research Fellowship (DGE-1747503). B.L.S. was supported in part by an NIH Ruth Kirschstein National Research Service Award (F32 Al138918). The authors made use of facilities at the UW-Madison including the Biophysics Instrumentation Facility (supported by the NSF (BIR-9512577) and NIH (S10 RR13790)) and at the UW-Madison Paul Bender Chemical Instrument Center.

\section{AUTHOR CONTRIBUTIONS}

H.E.B., M.E.B., M.J.S., and M.E.M. conceived of and designed this project. M.J.S., M.A.M., M.E.M., and M.E.B. designed and synthesized the probe molecules. M.J.S., M.E.B., M.A.M., and E.E.S. performed all biological assays. M.J.S. and B.L.S. cloned plasmids. All authors 
participated in the analysis of the data. M.J.S., M.E.B., and H.E.B. wrote the manuscript, and all authors contributed to the editing of the manuscript.

\section{COMPETING INTERESTS}

The authors declare no competing interests.

\section{ORCID $^{\circledR}$ IDs}

Matthew J. Styles - https://orcid.org/0000-0003-3236-0425

Helen E. Blackwell - https://orcid.org/0000-0003-4261-8194

\section{REFERENCES}

$1 \quad$ Miller, M. B. \& Bassler, B. L. Quorum sensing in bacteria. Annu Rev Microbio/ 55, 165199, (2001).

2 Fuqua, C. \& Greenberg, E. P. Listening in on bacteria: acyl-homoserine lactone signalling. Nat Rev Mol Cell Biol 3, 685, (2002).

3 Churchill, M. E. A. \& Chen, L. Structural Basis of Acyl-homoserine Lactone-Dependent Signaling. Chem Rev 111, 68-85, (2011).

4 Welsh, M. A. \& Blackwell, H. E. Chemical probes of quorum sensing: from compound development to biological discovery. FEMS Microbiol Rev 40, 774-794, (2016).

$5 \quad$ Whiteley, M., Diggle, S. P. \& Greenberg, E. P. Progress in and promise of bacterial quorum sensing research. Nature 551, 313, (2017).

6 Geske, G. D., O'Neill, J. C., Miller, D. M., Mattmann, M. E. \& Blackwell, H. E. Modulation of bacterial quorum sensing with synthetic ligands: systematic evaluation of $\mathrm{N}$-acylated homoserine lactones in multiple species and new insights into their mechanisms of action. JACS 129, 13613-13625, (2007).

7 Swem, L. R. et al. A quorum-sensing antagonist targets both membrane-bound and cytoplasmic receptors and controls bacterial pathogenicity. Mol Cell 35, 143-153, (2009).

8 Amara, N. et al. Covalent inhibition of bacterial quorum sensing. JACS 131, 1061010619, (2009). 
9 Galloway, W. R., Hodgkinson, J. T., Bowden, S., Welch, M. \& Spring, D. R. Applications of small molecule activators and inhibitors of quorum sensing in Gram-negative bacteria. Trends Microbiol 20, 449-458, (2012).

10 O'Loughlin, C. T. et al. A quorum-sensing inhibitor blocks Pseudomonas aeruginosa virulence and biofilm formation. PNAS 110, 17981-17986, (2013).

11 Manson, D. E., O'Reilly, M. C., Nyffeler, K. E. \& Blackwell, H. E. Design, Synthesis, and Biochemical Characterization of Non-Native Antagonists of the Pseudomonas aeruginosa Quorum Sensing Receptor LasR with Nanomolar IC50 Values. ACS Inf Dis 6, 649-661, (2020).

12 Styles, M. J. et al. Chemical Control of Quorum Sensing in E. coli: Identification of Small Molecule Modulators of SdiA and Mechanistic Characterization of a Covalent Inhibitor. ACS Inf Dis 6, 3092-3103, (2020).

13 Boursier, M. E., Manson, D. E., Combs, J. B. \& Blackwell, H. E. A comparative study of non-native $\mathrm{N}$-acyl L-homoserine lactone analogs in two Pseudomonas aeruginosa quorum sensing receptors that share a common native ligand yet inversely regulate virulence. Bioorg Med Chem 26, 5336-5342, (2018).

14 Mclnnis, C. E. \& Blackwell, H. E. Thiolactone modulators of quorum sensing revealed through library design and screening. Bioorg Med Chem 19, 4820-4828, (2011).

15 Boursier, M. E., Combs, J. B. \& Blackwell, H. E. N-Acyl L-Homocysteine Thiolactones Are Potent and Stable Synthetic Modulators of the RhIR Quorum Sensing Receptor in Pseudomonas aeruginosa. ACS Chem Biol 14, 186-191, (2019).

16 Zhu, J. \& Winans, S. C. Autoinducer binding by the quorum-sensing regulator TraR increases affinity for target promoters in vitro and decreases TraR turnover rates in whole cells. PNAS 96, 4832-4837, (1999).

17 Bottomley, M. J., Muraglia, E., Bazzo, R. \& Carfi, A. Molecular insights into quorum sensing in the human pathogen Pseudomonas aeruginosa from the structure of the virulence regulator LasR bound to its autoinducer. J Biol Chem 282, 13592-13600, (2007).

18 Lintz, M. J., Oinuma, K.-I., Wysoczynski, C. L., Greenberg, E. P. \& Churchill, M. E. A. Crystal structure of QscR, a Pseudomonas aeruginosa quorum sensing signal receptor. PNAS 108, 15763-15768, (2011).

19 Schaefer, A. L., Hanzelka, B. L., Eberhard, A. \& Greenberg, E. P. Quorum sensing in Vibrio fischeri: probing autoinducer-LuxR interactions with autoinducer analogs. $J$ Bacteriol 178, 2897-2901, (1996).

20 Schuster, M., Urbanowski, M. L. \& Greenberg, E. P. Promoter specificity in Pseudomonas aeruginosa quorum sensing revealed by DNA binding of purified LasR. PNAS 101, 15833-15839, (2004). 
21 Oinuma, K.-I. \& Greenberg, E. P. Acyl-Homoserine Lactone Binding to and Stability of the Orphan Pseudomonas aeruginosa Quorum-Sensing Signal Receptor QscR. J Bacteriol 193, 421, (2011).

$22 \mathrm{Kim}, \mathrm{T}$. et al. Structural insights into the molecular mechanism of Escherichia coli SdiA, a quorum-sensing receptor. Acta Crystallogr D Biol Crystallogr 70, 694-707, (2014).

23 Poulter, S., Carlton, T. M., Spring, D. R. \& Salmond, G. P. The Serratia LuxR family regulator CarR 39006 activates transcription independently of cognate quorum sensing signals. Mol Microbiol 80, 1120-1131, (2011).

24 Welch, M. et al. N-acyl homoserine lactone binding to the CarR receptor determines quorum-sensing specificity in Erwinia. Embo J 19, 631-641, (2000).

25 Wu, P. \& Brand, L. Resonance energy transfer: methods and applications. Anal Biochem 218, 1-13, (1994).

26 Lee, M. M. \& Peterson, B. R. Quantification of Small Molecule-Protein Interactions using FRET between Tryptophan and the Pacific Blue Fluorophore. ACS Omega 1, 1266-1276, (2016).

27 Mattmann, M. E., Shipway, P. M., Heth, N. J. \& Blackwell, H. E. Potent and selective synthetic modulators of a quorum sensing repressor in Pseudomonas aeruginosa identified from second-generation libraries of $\mathrm{N}$-acylated $\mathrm{L}$-homoserine lactones. Chembiochem 12, 942-949, (2011).

28 Eibergen, N. R., Moore, J. D., Mattmann, M. E. \& Blackwell, H. E. Potent and Selective Modulation of the RhIR Quorum Sensing Receptor by Using Non-native Ligands: An Emerging Target for Virulence Control in Pseudomonas aeruginosa. Chembiochem 16, 2348-2356, (2015).

29 Slinger, B. L., Deay, J. J., Chandler, J. R. \& Blackwell, H. E. Potent modulation of the CepR quorum sensing receptor and virulence in a Burkholderia cepacia complex member using non-native lactone ligands. Sci Rep 9, 13449, (2019).

30 Styles, M. J. \& Blackwell, H. E. Non-native autoinducer analogs capable of modulating the SdiA quorum sensing receptor in Salmonella enterica serovar Typhimurium. Beilstein J Org Chem 14, 2651-2664, (2018).

31 Gomes, J., Huber, N., Grunau, A., Eberl, L. \& Gademann, K. Fluorescent Labeling Agents for Quorum-Sensing Receptors (FLAQS) in Live Cells. Chem Eur J 19, 97669770, (2013).

32 Wysoczynski-Horita, C. L. et al. Mechanism of agonism and antagonism of the Pseudomonas aeruginosa quorum sensing regulator QscR with non-native ligands. Mol Microbiol 108, 240-257, (2018).

33 Gerdt, J. P., Mclnnis, C. E., Schell, T. L., Rossi, F. M. \& Blackwell, H. E. Mutational analysis of the quorum-sensing receptor LasR reveals interactions that govern activation and inhibition by nonlactone ligands. Chem Biol 21, 1361-1369, (2014). 
34 Tonge, P. J. Quantifying the Interactions between Biomolecules: Guidelines for Assay Design and Data Analysis. ACS Inf Dis 5, 796-808, (2019).

35 Pollard, T. D. A guide to simple and informative binding assays. Mol Biol Cell 21, 40614067, (2010).

36 Zhang, J. H., Chung, T. D. \& Oldenburg, K. R. A Simple Statistical Parameter for Use in Evaluation and Validation of High Throughput Screening Assays. J Biomol Screen 4, 6773, (1999).

37 O'Reilly, M. C. et al. Structural and Biochemical Studies of Non-native Agonists of the LasR Quorum-Sensing Receptor Reveal an L3 Loop "Out" Conformation for LasR. Cell Chem Biol 25, 1128-1139 (2018).

38 Moore, J. D., Rossi, F. M., Welsh, M. A., Nyffeler, K. E. \& Blackwell, H. E. A Comparative Analysis of Synthetic Quorum Sensing Modulators in Pseudomonas aeruginosa: New Insights into Mechanism, Active Efflux Susceptibility, Phenotypic Response, and NextGeneration Ligand Design. JACS 137, 14626-14639, (2015).

39 Boursier, M. E. et al. Structure-Function Analyses of the N-Butanoyl L-Homoserine Lactone Quorum-Sensing Signal Define Features Critical to Activity in RhIR. ACS Chem Biol 13, 2655-2662, (2018).

40 Zarkan, A., Liu, J., Matuszewska, M., Gaimster, H. \& Summers, D. K. Local and Universal Action: The Paradoxes of Indole Signalling in Bacteria. Trends Microbiol 28, 566-577, (2020).

41 Lee, J., Jayaraman, A. \& Wood, T. K. Indole is an inter-species biofilm signal mediated by SdiA. BMC Microbiol 7, 42, (2007).

42 Chu, W. et al. Indole production promotes Escherichia coli mixed-culture growth with Pseudomonas aeruginosa by inhibiting quorum signaling. Appl Environ Microbiol 78, 411419, (2012).

43 Hidalgo-Romano, B. et al. Indole inhibition of N-acylated homoserine lactone-mediated quorum signalling is widespread in Gram-negative bacteria. Microbiology 160, 24642473, (2014).

44 Sabag-Daigle, A., Soares, J. A., Smith, J. N., Elmasry, M. E. \& Ahmer, B. M. The acyl homoserine lactone receptor, SdiA, of Escherichia coli and Salmonella enterica serovar Typhimurium does not respond to indole. Appl Environ Microbiol 78, 5424-5431, (2012).

45 Kim, J. \& Park, W. Indole inhibits bacterial quorum sensing signal transmission by interfering with quorum sensing regulator folding. Microbiology 159, 2616-2625, (2013).

$46 \mathrm{Wu}, \mathrm{H}$. et al. Synthetic furanones inhibit quorum-sensing and enhance bacterial clearance in Pseudomonas aeruginosa lung infection in mice. J Antimicrob Chemother 53, 1054-1061, (2004).

47 Müh, U. et al. Novel Pseudomonas aeruginosa quorum-sensing inhibitors identified in an ultra-high-throughput screen. Antimicrob Agents Chemother 50, 3674-3679, (2006). 
48 Zou, Y. \& Nair, S. K. Molecular basis for the recognition of structurally distinct autoinducer mimics by the Pseudomonas aeruginosa LasR quorum-sensing signaling receptor. Chem Biol 16, 961-970, (2009).

49 Venturi, V. \& Ahmer, B. M. Editorial: LuxR Solos are Becoming Major Players in Cell-Cell Communication in Bacteria. Front Cell Infect Microbiol 5, 89, (2015).

50 Hudaiberdiev, S. et al. Census of solo LuxR genes in prokaryotic genomes. Front Cell Infect Microbiol 5, 20, (2015). 